\title{
Development of the Monitoring System of Plasma Behavior Using a CCD Camera in the GAMMA 10 Tandem Mirror
}

\author{
Hirokazu KAWANO, Yousuke NAKASHIMA, Yuta HIGASHIZONO, Nobuhiro NISHINO ${ }^{1}$, \\ Takayuki KOBAYASHI, Yoshiyuki MISHIMA, Mirai SUZUKI, Keiji MORI, Yusuke KUBOTA, \\ Masashi YOSHIKAWA and Teruji CHO \\ Plasma Research Center, University of Tsukuba, Tsukuba, Ibaraki 305-8577, Japan \\ ${ }^{1}$ Graduate school of Engineering, Hiroshima University, Hiroshima 739-8527, Japan
}

(Received 4 December 2006 / Accepted 6 March 2007)

\begin{abstract}
In the central-cell of the GAMMA 10 tandem mirror, a medium-speed camera (CCD camera, 400 frames per second, $216 \times 640$ pixel) has been installed for the observation of plasma behavior. This camera system is designed for monitoring the plasma position and movement in the whole discharge duration. The captured twodimensional (2-D) images are automatically displayed just after the plasma shot and stored sequentially shot by shot. This system has been established as a helpful tool for optimizing the plasma production and heating systems by measuring the plasma behavior in several experimental conditions. The camera system shows that the intensity of the visible light emission on the central-cell limiter accompanied by central electron cyclotron heating (C-ECH) correlate with the wall conditioning and immersion length of a movable limiter (iris limiter) in the central cell.
\end{abstract}

(C) 2007 The Japan Society of Plasma Science and Nuclear Fusion Research

Keywords: GAMMA 10, tandem mirror, visible-light emission, two-dimensional image, CCD camera, plasma monitoring system, limiter-plasma interaction

DOI: $10.1585 /$ pfr.2.S1126

\section{Introduction}

High-speed camera is one of very useful tools to observe precise plasma behavior and can capture the instantaneous two-dimensional (2-D) structure of its visible emission. 2-D image measurements have been successfully demonstrated in many fusion experimental devices around the world with the development of camera performance [1-3]. 2-D image of plasma behavior also gives us a lot of useful information for controlling the plasma position and understanding the plasma response on plasma heating systems. In GAMMA 10 tandem mirror plasmas, investigation of hydrogen recycling and edge plasma behavior has been extensively performed on the basis of $\mathrm{H} \alpha$ intensity and Langmuir probe measurements [4-7]. In April 2004, based on the bi-directional collaboration program, measurements of edge plasma fluctuation by using a highspeed camera was started in the central-cell and the plasma motion has been analyzed based on the detailed time behavior of 2-D images [8-11]. Recently, a CCD camera system was newly designed for monitoring the plasma position and movement in the whole discharge duration. In this paper, the detail of the monitoring system is described and the observed results are discussed from the response of electron cyclotron heating $(\mathrm{C}-\mathrm{ECH})$ by using a gyrotron installed in the central-cell.

\section{GAMMA 10 and Plasma Monitor- ing System}

Figure 1 shows the schematic view of GAMMA 10 and the experimental setup used in this study. $Z$-axis is defined along the direction of the magnetic field. GAMMA 10 is aligned along the east ( $Z$ is negative.) and west ( $Z$ is positive.). $Z=0 \mathrm{~cm}$ is at the center of the central cell. Plasmas are produced by two plasma guns from both ends and then built up with two ion cyclotron range of frequency

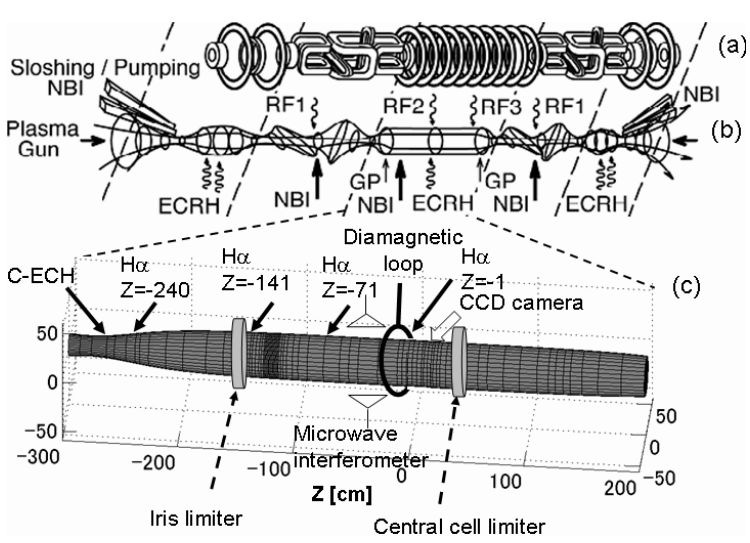

Fig. 1 Schematic view of the experimental setup. (a) Coil arrangement, (b) magnetic flux tube and the location of plasma production and heating systems, (c) detailed geometry and diagnostics in the central-cell. 
(ICRF) systems. Two types of gas puffers have been used to maintain the plasma. In addition to ICRF heating, C$\mathrm{ECH}$ is installed to heat electrons of the central-cell in the course of the discharge. $\mathrm{H} \alpha$ line emission detectors $(Z=-1,-71,-141 \mathrm{~cm})$ and a CCD camera $(Z=0 \mathrm{~cm})$ are installed along the machine axis vertical to the magnetic field [5]. A microwave interferometer and a diamagnetic loop are installed near the central-cell mid-plane to measure the electron line density (NLcc) and the diamagnetism (DMcc) in the central-cell. A central-cell limiter $(Z=+30 \mathrm{~cm})$ and an iris limiter $(Z=-155 \mathrm{~cm})$ are located in the central-cell and define the plasma boundary.

Figure 2 shows the schematic view of the monitoring system. The monitoring system consists of a CCD camera and two PCs. One PC is set in the GAMMA 10 machine room, and the other is in the operating room. The maximum framing speed of the camera is 400 frames/s. Through a horizontal port at the central-cell mid-plane, an area of approximately $40 \mathrm{~cm} \times 120 \mathrm{~cm}$ at the machine axis is imaged onto the $216 \times 640$ pixel. The monitoring system is operated according to the following sequence: First, the CCD camera, directly connected to the PC by way of an interface board with PCI bus, starts to record the visible image of plasma receiving the trigger pulse. After capturing

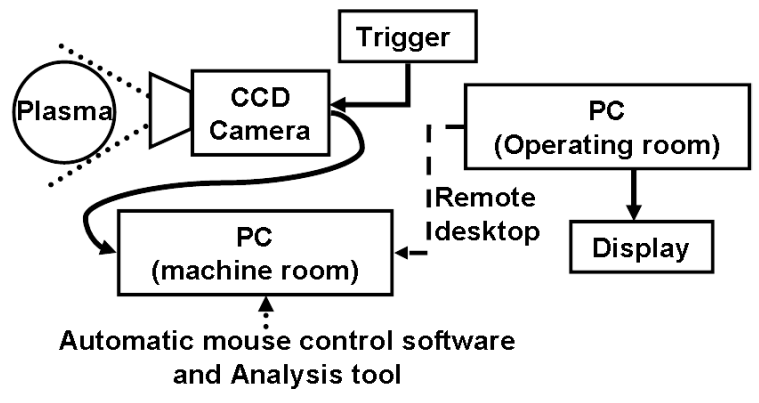

Fig. 2 Schematic diagram of the monitoring system.

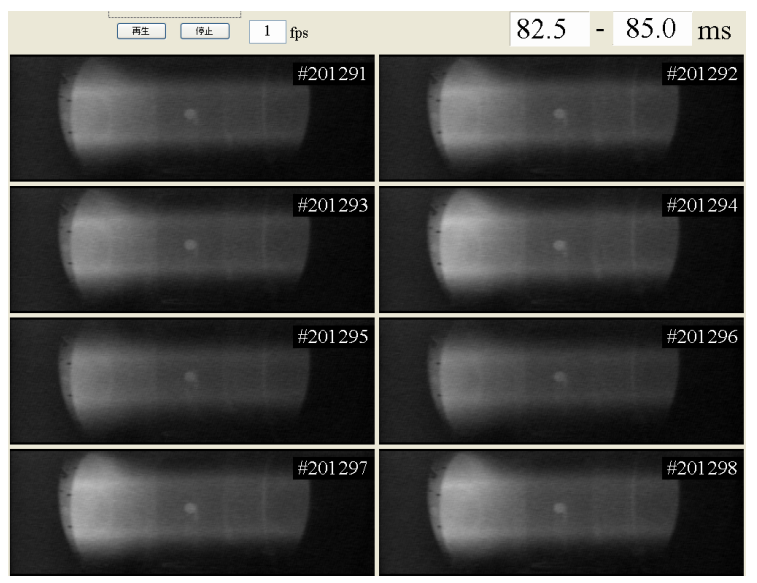

Fig. 3 Images on the desktop screen by this monitoring system. This is the screen of an analysis mode. 8 shots are displayed simultaneously in the analysis mode and 10 shots are displayed in the monitor mode. the picture images by the given frame number, the image data are transferred to a hard disc in the PC and are labeled with the corresponding shot number and then it returns to the trigger-waiting mode. The above procedure is executed by automatic mouse control software, and the data are displayed as a movie in shot-to-shot intervals and latest 10 shots are displayed simultaneously by the help of a data analysis tool. An example of captured screen-view of the $\mathrm{PC}$ in the operating room is shown in Fig. 3. Therefore, the changes of the plasma behavior can be figured out instantaneously. By way of this remote control system, we can watch and handle the PC in the machine room from the PC in the operating room.

\section{Experimental Results and Discus- sion}

\subsection{Time behavior of plasma parameter and 2-D visible images captured with a CCD camera}

Figure 4 shows an example of the time behavior of DMcc, NLcc and $\mathrm{H} \alpha$ intensity of a standard ICRF-heated plasma measured at the central-cell. In this experiment, plug ECH is injected from $t=160 \mathrm{~ms}$ to $t=166 \mathrm{~ms}$ and barrier ECH is from $t=160 \mathrm{~ms}$ to $t=180 \mathrm{~ms}$. C-ECH is applied from $t=160 \mathrm{~ms}$ to $t=166 \mathrm{~ms}$ and resultant DMcc is slightly reduced at the onset of $\mathrm{C}-\mathrm{ECH}$. On the other hand, $\mathrm{H} \alpha$ intensity increases during $\mathrm{C}-\mathrm{ECH}$. Figure 5 shows the time evolution of the 2-D image of the visible emission
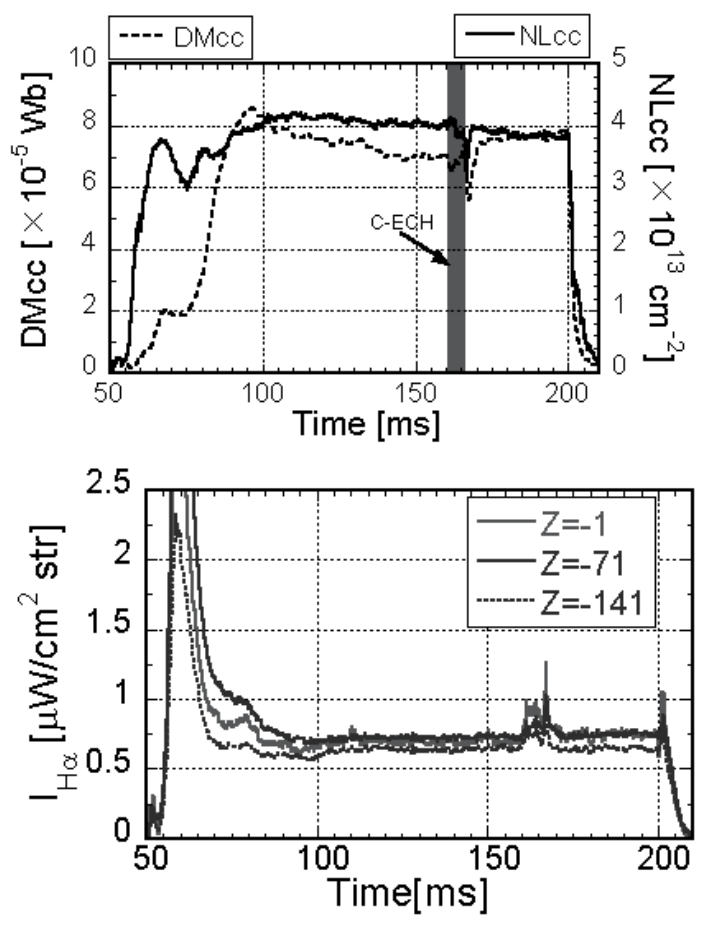

Fig. 4 Time behavior of plasma parameters; Diamagnetism (DMcc), electron line density (NLcc) and $\mathrm{H} \alpha$ line intensity at various locations in the central-cell. 
from the plasma observed by this monitoring system. The central-cell limiter $(360 \mathrm{~mm}$ in diameter) and the plasma are observed in these images. It is seen that the interaction with plasma and the limiter affects the light emission. The position and the motion of the plasma and the intensity of the emission are monitored by this system. At the initial stage of the plasma discharge, the plasma diameter is slender. The plasma becomes fat with the increase of NLcc. After plasma build-up is completed, the light emission is observed on the central-cell limiter. Especially, the emission from the top of the limiter becomes much strong when $\mathrm{C}-\mathrm{ECH}$ is injected. It is thought that the emission comes from impurity or the recycling of hydrogen near the wall and the limiter in the central-cell. It is because that plasma diameter is bigger or plasma move upward during the C-ECH injection. The strong light emission is observed again when the plasma collapses.

\subsection{Effects of C-ECH on the light emission}

As shown in Fig. 5 (d), the light emission from the top of the central-cell limiter is strong during the $\mathrm{C}-\mathrm{ECH}$ injection. In Fig. 6, the light emission intensity on the area just below the central-cell limiter shown in Fig. 5 (c) (dashed line) is compared with that of Fig. 5 (d). Although the light emission intensity with $\mathrm{C}-\mathrm{ECH}$ from the pixel position in 20 to 45 is saturated in the CCD camera, it is clear that the intensity with $\mathrm{C}-\mathrm{ECH}$ is stronger than that without $\mathrm{C}-\mathrm{ECH}$. On the other hand, the intensity without $\mathrm{C}-\mathrm{ECH}$ is stronger than that with $\mathrm{C}-\mathrm{ECH}$ at the bottom of the central-cell (pixel position from 150 to 200). Therefore, it is thought that the plasma moves little upward during the $\mathrm{C}-\mathrm{ECH}$ injection.

In this experiment, the iris limiter is used for controlling the limiter-plasma interaction at the central-cell limiter as mentioned above. Figure 7 shows the images during
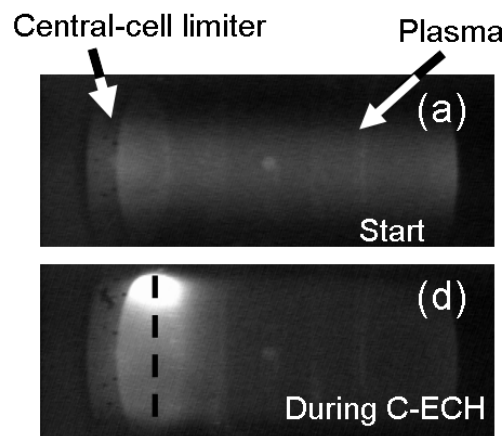
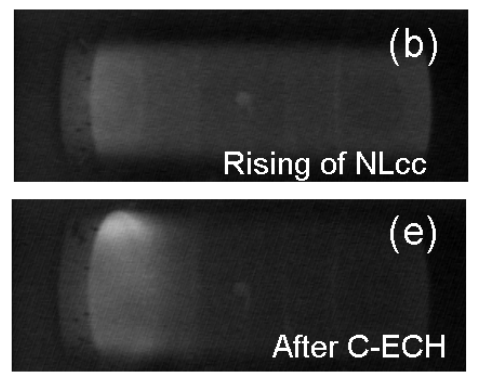
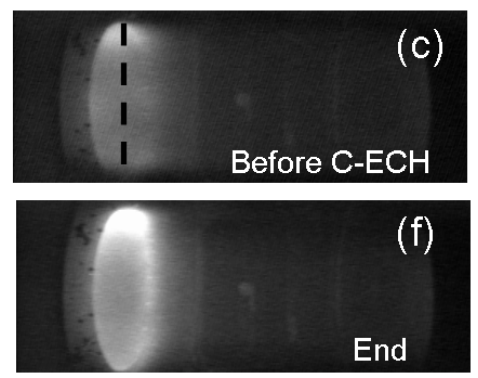

Fig. 5 Time evolution of the visible emission from the plasma observed by this monitoring system.

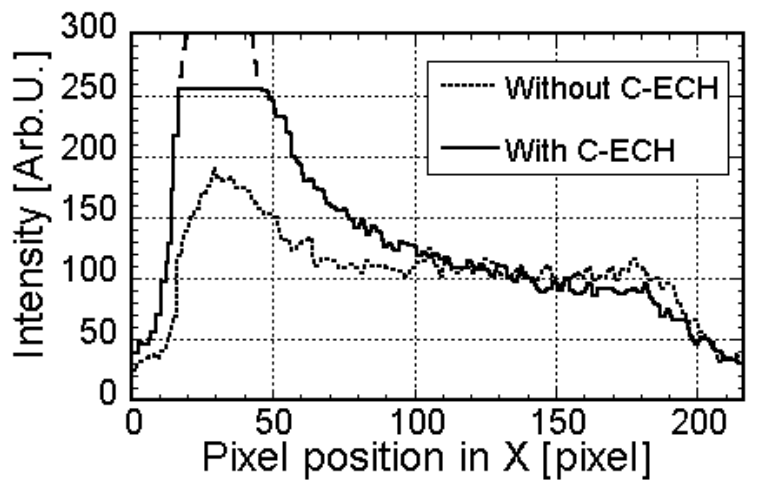

Fig. 6 Comparison between the light emission intensity on the area just below the central-cell limiter (dashed line) of Fig. 5 (c) and that of Fig. 5 (d).
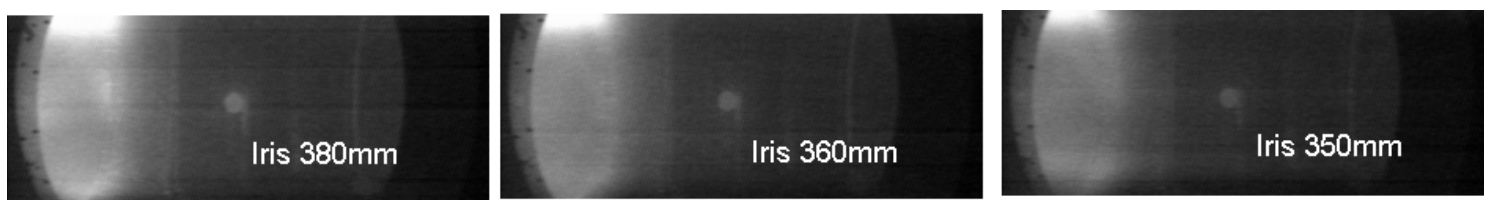

Fig. 7 Images during the C-ECH injection when the insertion of iris limiter is $380,360,350 \mathrm{~mm}$. 


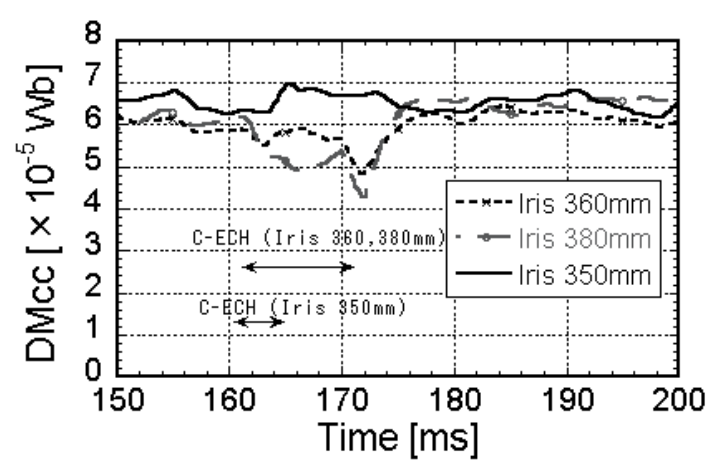

Fig. 8 Time behavior of the DMcc in three shots (iris $=380$, $360,350 \mathrm{~mm})$.

$\mathrm{C}-\mathrm{ECH}$ injection when the diameter of the iris limiter is 380, 360, and $350 \mathrm{~mm}$. As shown in Fig. 7, the intensity of the light emission at the top of the central-cell limiter decreases according to the insertion of the iris limiter. Figure 8 shows the time behavior of the DMcc of three shots with different radius of the iris limiter. The extent of reduction in DMcc is also improved. It is thought that the plasma surface is scraped with inserting the iris limiter and the edge plasma does not heat up, then the plasma does not interact with the central-cell limiter very much and the light emission of the limiter decreases during the C-ECH injection.

\section{Conclusion}

The monitoring system of plasma behavior using a medium-speed CCD camera (400 fps) has been successfully built up in the GAMMA 10 tandem mirror.
This system gives us a lot of information from the plasmas. The conditioning and the position of the plasma are monitored quickly, and then their feedback can be used to optimize the plasma performance. From the 2-D image analyses, it is clarified that the strong light emission on the central limiter during the $\mathrm{C}-\mathrm{ECH}$ is related to the abrupt reduction of diamagnetism. Adequate insertion of the iris limiter is also proved to improve the reduction of DMcc. Further investigation with an improved imaging system to see the iris limiter directly will be possible to understand the relation between iris limiter and DMcc.

\section{Acknowledgments}

The authors would like to thank the members of the GAMMA 10 group in University of Tsukuba for their help in the experiment.

[1] R.J. Maqueda and G.A. Wurden et al., Rev. Sci. Instrum. 74(3), 2020 (2003).

[2] S.J. Zweben et al., Phys. Plasmas 9, 1981 (2002).

[3] N. Nishino et al., J. Nucl. Mater. 337-339, 1073 (2005).

[4] Y. Nakashima et al., J.Nucl. Mater. 196-198, 493 (1992).

[5] Y. Nakashima et al., J.Nucl. Mater. 241-243, 1011 (1997).

[6] Y. Nakashima et al., J. Plasma Fusion Res. SERIES 5, 428 (2002).

[7] H. Higaki et al., Rev. Sci. Instrum 75, 4085 (2004).

[8] Y. Nakashima et al., to be published in J. Nucl. Mater. (2007).

[9] N. Nishino et al., J. Plasma Fusion Res. 1, 035 (2006).

[10] H.Higaki et al., to be published in Tans. Fusion Sci. and Technical (2007).

[11] Y. Nakashima et al., ibid. 